\title{
Back from the dead; the curious tale of the predatory cyanobacterium Vampirovibrio chlorellavorus
}

Rochelle M Soo, Ben J Woodcroft, Donovan H Parks, Gene W Tyson, Philip Hugenholtz

An uncultured non-photosynthetic basal lineage of the Cyanobacteria, the Melainabacteria, was recently characterised by metagenomic analyses of aphotic environmental samples. However, a predatory bacterium, Vampirovibrio chlorellavorus, originally described in 1972 appears to be the first cultured representative of the Melainabacteria based on a 16S rRNA sequence recovered from a lyophilised co-culture of the organism. Here, we sequenced the genome of $V$. chlorellavorus directly from 36 year-old lyophilised material that could not be resuscitated confirming its identity as a member of the Melainabacteria. We identified attributes in the genome that likely allow $V$. chlorellavorus to function as an obligate predator of the microalga Chlorella vulgaris, and predict that it is the first described predator to use an Agrobacterium tumefaciens-like conjugative type IV secretion system to invade its host. $V$. chlorellavorus is the first cyanobacterium recognised to have a predatory lifestyle and further supports the assertion that Melainabacteria are nonphotosynthetic. 
2 Rochelle M. Soo ${ }^{1}$, Ben J. Woodcroft ${ }^{1}$, Donovan H. Parks ${ }^{1}$, Gene W. Tyson ${ }^{1,2}$ and Philip

3 Hugenholtz ${ }^{1,3 *}$

4

5 'Australian Centre for Ecogenomics, School of Chemistry and Molecular Biosciences, The University of

6 Queensland, St Lucia, QLD 4072, Australia

7 2Advanced Water Management Centre, The University of Queensland, St Lucia, QLD 4072, Australia

8 IInstitute for Molecular Bioscience, The University of Queensland, St Lucia, QLD 4072, Australia

9

$10 *$ Corresponding author:

11 Philip Hugenholtz

12 Australian Centre for Ecogenomics, School of Chemistry and Molecular Biosciences, The University of

13 Queensland, St Lucia, QLD 4072, Australia

$14+61733653822$

15 p.hugenholtz@uq.edu.au

16 


\section{Introduction}

18 Predatory microorganisms attack and digest their prey, which can be either bacteria or microbial

19 eukaryotes (Coder \& Starr, 1978; Stolp \& Starr,1963). They have been found in a range of

20 environments, including terrestrial, freshwater, estuaries, oceans, sewages and animal faeces (Jurkevitch,

21 2007). Microbial predators have been classified as obligate (unable to grow in the absence of prey) or

22 facultative (able to grow as a pure culture without the presence of prey). In addition they can be

23 periplasmic (penetrate and attach to the inner membrane), epibiotic (attach to the outside), endobiotic

24 (penetrate the cytoplasm) or wolf-pack (swarming as a 'wolf-pack' towards prey, which they kill and

25 degrade) (Pasternak et al., 2013; Velicer et al., 2000). To date, four bacterial phyla harbour microbial

26 predators; the Proteobacteria, Actinobacteria, Bacteroidetes and Chloroflexi (Casida, 1983; Kiss et al.,

27 2011; Saw et al., 2012; Stolp \& Starr, 1963).

28

29 In 1972, Gromov and Mamkaeva first described the predatory nature of Bdellovibrio chlorellavorus

30 towards the microalgae Chlorella vulgaris in a Ukrainian freshwater reservoir (Gromov \& Mamkaeva,

31 1972). They reported that co-inoculation of the alga and bacterium resulted in clumping and colour

32 change of algal cells, formation of refractile bodies and finally algal cell death. However, unlike other

33 Bdellovibrio species that invade the periplasm of Gram-negative bacteria, B. chlorellavorus only

34 attached to the surface of $C$. vulgaris, producing peripheral vacuoles in the alga followed by a gradual

35 dissolution of the infected cell contents (Coder \& Goff, 1986). This distinct mode of predation called

36 into question the classification of B. chlorellavorus as a Bdellovibrio (Coder \& Starr, 1978) resulting in

37 its reclassification as Vampirovibrio chlorellavorus in 1980, although its higher level assignment to the

38 Deltaproteobacteria was retained (Gromov \& Mamkaeva, 1980). 
40 Co-cultures of $V$. chlorellavorus and C. vulgaris were deposited in three culture collections in 1978

41 (Coder \& Starr, 1978). However, to the best of our knowledge there are no reports of successful

42 resuscitation of the organism from lyophilised material. The only subsequent studies of $V$.

43 chlorellavorus were based on co-cultures obtained directly from the investigators who originally

44 enriched the bacterium (Coder \& Goff, 1986; Mamkaeva \& Rybal'chenko, 1979). The American Type

45 Culture Collection (ATCC) was able to successfully extract DNA from one of the 32 year-old

46 lyophilised co-cultures and sequence the 16S rRNA gene of $V$. chlorellavorus (Genbank acc. no.

47 HM038000). Comparative analyses of this sequence indicate that $V$. chlorellavorus is actually a member

48 of the phylum Cyanobacteria rather than the Proteobacteria according to the Greengenes (McDonald et

49 al., 2012) and Silva (Quast et al., 2013) taxonomies. This may explain why the culture could not be

50 revived as Cyanobacteria are notoriously difficult to resuscitate from lyophilised material (Corbett \&

51 Parker, 1976). More specifically, $V$. chlorellavorus is a member of a recently described basal lineage of

52 non-photosynthetic Cyanobacteria, the class Melainabacteria ((Soo et al., 2014), originally classified as a

53 separate phylum (Di Rienzi et al., 2013)). Here, we report the near-complete genome of $V$.

54 chlorellavorus sequenced directly from a 36-year-old vial of co-cultured lyophilised cells, confirm its

55 phylogenetic position in the Cyanobacteria, and infer the molecular underpinnings of its predatory life 56 cycle.

57

58 


\section{Materials and Methods}

\section{Sample collection}

61 Co-cultured Vampirovibrio chlorellavorus and Chlorella vulgaris (NCIB 11383) (deposited in 1978 by

62 Coder and Starr) were obtained as lyophilised cells from the National Collections of Industrial and

63 Marine Bacteria (NCIMB), Aberdeen, Scotland.

64

\section{Genomic DNA extraction}

66 Genomic DNA (gDNA) was extracted from lyophilised cells using a MoBio Soil Extraction kit (MoBio

67 Laboratories, Carlsbad, CA). gDNA was quantified using a Qubit 2.0 fluorometer (Life technologies).

68 1ng of the gDNA was used to construct a paired-end library with the Illumina Nextera XT DNA Sample

69 Preparation kit according to protocol but with double size selection to obtain an insert size of 300-800

70 bp (Quail et al., 2009). The library was sequenced on an Illumina Miseq system using the Miseq Reagent

71 Kit v3 at the Institute of Molecular Bioscience, University of Queensland.

72

\section{Genome assembly, completeness and contamination}

74 Sequencing reads were processed with FastQC to check for quality (Andrews, 2010) and Illumina

75 Nextera adaptors were removed using FASTX-Toolkit (http://hannonlab.cshl.edu/fastx_toolkit/). Reads

76 were parsed through GraftM (https:/github.com/geronimp/graftM) version r2439db using the May, 2013

77 version of the Greengenes database 97\% OTUs (operational taxonomic units) as a reference (McDonald

78 et al., 2012) to identify those containing parts of $16 \mathrm{~S}$ or $18 \mathrm{~S}$ rRNA genes using default parameters. The

795 ' end of all reads was trimmed ( 20bp) to remove low-quality sequence and paired reads were

80 assembled into contigs with a kmer size of 63 using CLC Genomics Workbench v7.0 (CLC bio). The

81 statistical package R with ggplot2 (Wickham, 2009) was used to plot GC content against coverage 
82 allowing contigs belonging to the $V$. chlorellavorus genome to be identified. A discrete cluster of contigs

83 with $>180 \mathrm{x}$ coverage and a GC range of $42-54 \%$ were identified as belonging to $V$. chlorellavorus, while

84 contigs with $<180 x$ coverage were assigned to $C$. vulgaris (Fig. S1). BLASTN (Altschul et al., 1990)

85 (v2.2.29+) using default settings was used to verify that contigs with $>180 \mathrm{x}$ coverage had homology to

86 bacterial sequences with NCBI's non-redundant database. Additionally, the 16S rRNA gene was

87 identified using Prokka v1.8 (Seemann, 2014) and a BLASTN search was used to identify the closest

88 neighbour in the May, 2013 version of the Greengenes database (McDonald et al., 2012). The

89 completeness and contamination of the genome belonging to $V$. chlorellavorus was examined using

90 CheckM v0.9.5 (Parks et al., 2014a) with a set of 104 conserved bacterial single-copy marker genes (Soo

91 et al., 2014). IslandViewer was used to identify genomic islands (Langille \& Brinkman, 2009) with the

92 SIGI-HMM programme (Waack et al., 2006).

93

94 Plasmids were identified using the 'roundup' mode of FinishM git version 5664703

95 (https://github.com/wwood/finishm), using raw reads as input, a kmer length of $51 \mathrm{bp}$ and a coverage

96 cutoff of 15. A combination of manual inspection of the assembly graph generated using the 'visualise'

97 mode and automated assembly with the 'assemble' mode confirmed that the contig ends unambiguously

98 joined together (i.e. they joined together and to no other contig ends) and that the two plasmid contigs

99 originally assembled with CLC were otherwise free of mis-assemblies. Plasmids were also confirmed by

100 the annotation of multiple transfer (tra) genes by the Integrated Microbial Genomics Expert Review

101 (IMG/ER) system (see below).

\section{Genome annotation}

103 The $V$. chlorellavorus genome was submitted to IMG/ER for annotation (Markowitz et al., 2009). The $V$.

104 chlorellavorus genome has been deposited at JGI and NCBI [JGI IMG-ER:2600254900]. The genome

105 was also annotated with prokka v.1.8 (Seemann, 2014) and the Uniref 90 database (Suzek et al., 2007). 
106 KEGG maps (Kanehisa et al., 2004) and gene annotations were used to reconstruct the metabolism of

107 the $V$. chlorellavorus genome. Individual genes that were annotated as 'hypothetical protein' or had been

108 potentially misannotated based on the annotation of surrounding genes were further explored through

109 BLASTN searches against the NCBI-nr database. A metabolic cartoon was prepared in Adobe Illustrator

110 CS6.

111

112 The methyl-accepting chemotaxis proteins identified by IMG-ER were submitted to InterProScan5

113 (Jones et al., 2014) to determine chemotaxis protein domains. Putative genes were annotated with the

114 dbCAN web server (Yin et al., 2012) to identify glycoside hydrolases and checked against the IMG

115 annotations and BLAST results. The MEROPS server (Rawlings et al., 2014) was used to identify

116 putative peptidases in $V$. chlorellavorus using batch BLAST.

118 A Genbank file for $V$. chlorellavorus was generated through the xBASE website (Chaudhuri et al.,

119 2008). The ribosomal proteins, chaperones and transcriptional and translational proteins of $V$.

120 chlorellavorus were used as representatives of recognised highly expressed genes to identify other

121 putatively highly expressed genes in the genome using PHX (predicted highly expressed) analysis using

122 the standard genetic code (http://www.cmbl.uga.edu/software/phxpa.html; Bhaya et al., 2000; Karlin \&

123 Mrázek, 2000). Putatively horizontally transferred (alien) genes were identified by their atypical codon

124 usage from the genome average also using PHX analysis.

125

126 Phylogenetic tree

127 A bacterial genome tree was inferred in order to establish the phylogenetic relationship of the $V$.

128 chlorellavorus genome. A set of 5,449 bacterial genomes previously identified as being of exceptional

129 quality were used to establish a set of bacterial marker genes suitable for phylogenetic inference (Parks 
130 et al., 2014a). An initial set of 178 single copy genes present exactly once in $>90 \%$ of the trusted

131 genomes (found in $>90 \%$ of the genomes) was identified using the PFAM (Finn et al., 2014) and

132 TIGRFAM (Haft et al., 2003) annotations provided by the Integrated Microbial Genomes (IMG;

133 (Markowitz et al., 2014)). The same protein family may be represented in both PFAM and TIGRFAM.

134 Families from these two databases were considered redundant if they matched the same genes in $>90 \%$

135 of the trusted genomes, in which case preference was given to the TIGRFAM families. Genes present

136 multiple times within a genome were considered to have congruent phylogenetic histories if all copies of

137 the gene were situated within a single conspecific clade within its gene tree. From the 178 initial genes,

13869 were removed from consideration as they exhibited divergent phylogenetic histories in $>1 \%$ of the

139 trusted genomes (Table S1). The remaining 109 genes were identified across an expanded set of 7732

140 bacterial genomes, including all known Melainabacteria genomes along with an outgroup of 169

141 archaeal genomes using Prodigal v2.60 (Hyatt et al., 2012) to identify call genes and HMMER v3.1b1

142 (http://hmmer.janelia.org) to assign genes to PFAM and TIGRFAM families. Gene assignment was

143 performed using model specific cutoff values for both the PFAM (-cut_gc) and TIGRFAM (-cut_tc)

144 HMMs. For both the individual gene trees and concatenated genome tree, genes were aligned with

145 HMMER v3.1b1 and phylogenetic inference performed with FastTree v2.1.7 (Price et al., 2009) under

146 the WAG+GAMMA model. Support values for the bacterial genome tree were determined by applying

147 FastTree to 100 bootstrapped replicates (Felsenstein, 1985). The 16S rRNA gene tree was constructed as

148 previously described (Soo et al., 2014). Briefly, the 16S rRNA gene from V. chlorellavorus was aligned

149 to the standard Greengenes alignment with PyNAST (McDonald et al., 2012). Aligned sequences and a

150 Greengenes reference alignment, version gg_13_5 were imported into ARB and the $V$. chlorellavorus

151 sequence alignment was corrected using the ARB EDIT tool. Representative taxa $(>1,300 \mathrm{nt})$ were

152 selected for constructing the alignments, which were exported from ARB (Ludwig et al., 2004) with

153 Lane mask filtering. Neighbour joining trees were calculated from the mask alingments with LogDet 
154 distance estimation using PAUP*4.0 (Swofford \& Sullivan, 2003) with 100 bootstrap replicates.

155 Maximum parsimony trees were calculated using PAUP*4.0 (Swofford \& Sullivan, 2003) with 100

156 bootstrap replicates. Maximum likelihood trees were calculated from the masked alignments using the

157 Generalized Time-Reversible model with Gamma and I options in RAxML version 7.7.8 (Stamatakis,

158 2006) (raxmlHPC-PTHREADS -f a -k -x 12345 -p 12345 -N 100 -T 4 -m GTRGAMMAI). Bootstrap

159 resampling data (100 replicates) were generated with SEQBOOT in the phylip package (Felsenstein,

1601989 ) and used for 100 bootstrap resamplings. Generated trees were re-imported into ARB for

161 visualisation.

162

163 Phylogenetic trees for virB4 and fliI genes

164 VirB4 sequences were obtained from Guglielmini et al. 2012. The phylip file

165 (figure3_mafft_alignment.phy) obtained from the DRYAD database was converted to an HMM using

166 HMMer v3.1b1 (http://hmmer.janelia.org) and the VirB4 sequences from $V$. chlorellavorus was aligned

167 to the HMM. The aligned sequences were used to construct a phylogenetic tree with phyml (v3.1)

168 (Guindon et al., 2010) using default settings (Guglielmini et al., 2012).

169

170 The HMM for TIGR03496 (FliI_clade 1) was used to identify fliI genes from 2,256 finished genomes in

171 the IMG database $\mathrm{v} 4$ and the 12 Melainabacteria genomes, including V. chlorellavorus. A phylogenetic

172 tree of the fli genes was constructed using FastTree (version 2.1.7) with default settings (Price et al., 173 2009).

174

175 Comparison of $\boldsymbol{V}$. chlorellavorus to other predatory bacteria 
176 The presence of orthologues for differentiating predatory and non-predatory bacteria as described in

177 Pasternak et al. (2013) were identified in the V. chlorellavorus genome using BLASTP (Altschul et al.,

178 1990) against the OrthoMCL DB v4 (Chen et al., 2006) with an e-value threshold of 1e-5.

179

180 Comparison of $\boldsymbol{V}$. chlorellavorus to other Melainabacteria genomes

181 Eleven Melainabacteria genomes were compared to the $V$. chlorellavorus genome (Di Rienzi et al.,

182 2013; Soo et al., 2014). COG profiles were constructed using homology search between putative genes

183 predicted with Prodigal v2.60 (Hyatt et al., 2010) and the 2003 COG database (Tatusov et al., 2003).

184 Genes were assigned to COGs using BLASTP (v2.2.22) with an e-value threshold of 1e-2, an alignment

185 length threshold of $70 \%$ and a percent identity threshold of $30 \%$. The relative percentage of a COG

186 category was calculated in relation to the total number of putative genes predicted for each genome.

187 STAMP v2.0.8 (Parks et al., 2014b) was used to explore the resulting COG profiles and create summary 188 plots.

189

190 Results and Discussion

191 Genome summary

192 A total of $701.2 \mathrm{Mbp}$ of shotgun sequence data ( 2 x 300 bp paired-end Illumina) was obtained from

193 DNA extracted from a co-culture of Vampirovibrio chlorellavorus and Chlorella vulgaris (NCIB

194 11384). A search of the unassembled dataset for 16S rRNA sequences revealed 333 reads mapping to $V$.

195 chlorellavorus (16 chloroplast, 3 mitochondria). No matches to other microorganisms were identified.

196 Sequence reads were assembled into 113 contigs comprising $3.2 \mathrm{Mbp}$. Ordination of the data by GC

197 content and mapping read depth revealed a high coverage cluster of contigs comprising $\sim 94 \%$ of the data

198 (Fig. S1). These contigs were inferred to belong to $V$. chlorellavorus by the presence of a 16S rRNA 
199 gene on one of the contigs (see below) and low coverage contigs were inferred to belong to the $C$.

200 vulgaris by best matches to reference Chlorella genomes. Inspection of the assemblies showed no

201 evidence for microheterogeneity (SNPs, indels) in the $V$. chlorellavorus contigs suggesting that it was a

202 pure bacterial strain. After manual curation, the genome of $V$. chlorellavorus was represented by 26

203 contigs comprising a total of $2.91 \mathrm{Mbp}$ with an average GC content of $51.4 \%$ and two plasmids

204 comprising $\sim 72 \mathrm{Kbp}$ and $\sim 50 \mathrm{Kbp}$ were identified which contained genes for conjugative gene transfer

205 (see below). These plasmids had mapping coverage similar to the genomic contigs suggesting that they

206 are low-copy. The genome was estimated to be near-complete with low contamination according to

207 CheckM (Parks et al., 2014a) suggesting that the fraction of missed genes in contig gaps was minimal.

208 The protein coding density of the genome is $87.1 \%$ and predicted to encode 2,847 putative genes, 41

209 tRNA genes which represent all 20 amino acids and one rRNA operon (only the 16S and 23S rRNA

210 genes were identified). Approximately two thirds (69.9\%) of the putative genes can be assigned to a

211 putative function and half (53.2\%) can be assigned to a COG category. $V$. chlorellavorus contains 13

212 transposases and 18 genomic islands (genomic regions that are thought to have horizontal origins)

\section{3 (Table 1).}

214

\section{Phylogeny and taxonomy}

216 The 16S rRNA gene obtained from the draft genome is identical to the reference sequence for $V$.

217 chlorellavorus ATCC 29753 (acc. HM038000) and comparative analysis confirmed its placement as a

218 deep-branching member of the Cyanobacteria phylum within the class Melainabacteria and order

219 Vampirovibrionales (Soo et al., 2014; Fig. 1B). Importantly, a concatenated gene tree of 109 conserved

220 single copy genes produced a robust topology consistent with the 16S rRNA tree, also placing $V$.

221 chlorellavorus in the class Melainabacteria (Fig. 1A: Fig. S2). These phylogenetic inferences clearly 
222 indicate that $V$. chlorellavorus is not a member of the Deltaproteobacteria as first suggested (Gromov \&

223 Mamkaeva, 1972).

224

\section{Cell shape and envelope}

226 Microscopy studies revealed that $V$. chlorellavorus has a pleomorphic life cycle, being cocci during its 227 free-living phase and vibrioid once attached to its host (Coder \& Starr, 1978). The V. chlorellavorus 228 genome contains genes for the shape-determining protein ( $m r e B)$ and a key cell division protein $(f t s Z)$,

229 which have been shown to be necessary for the maintenance of cell shape in Caulobacter crescentus and 230 Eschericia coli (Divakaruni et al., 2007; Varma \& Young, 2009). The bacterium also contains the genes

231 indicative of a Gram-negative cell envelope including those for the production of lipopolysaccharide 232 (LPS), Lipid A and O-antigen (Beveridge, 1999). This is consistent with prior ultrastructural imaging of

233 V. chlorellavorus which showed this bacterium has a typical Gram-negative cell envelope (Coder \& 234 Starr, 1978). Interestingly, the genome also contains surface layer homology (SLH) domains, suggesting 235 that the cell has the capacity to produce an S-layer, although no such structures were observable in 236 transmission electron microscopy (TEM) images (Coder \& Starr, 1978; Mamkaeva \& Rybal'chenko, 237 1979). This does not preclude their presence, however, because the samples were not processed 238 optimally for S-layer visualisation; and under unfavourable laboratory cultivation conditions, the 239 formation of the S-layer may be lost (Sára \& Sleytr, 2000; Šmarda et al., 2002). S-layers have been

240 observed in at least 60 strains of Cyanobacteria (Šmarda et al., 2002) and SLH domains have also been 241 found in other Melainabacterial genomes.

\section{Core metabolism}

244 The $V$. chlorellavorus genome encodes a complete glycolysis pathway utilising glucose-6-phosphate, 245 glycerol and mannose, the pentose phosphate pathway and a tricarboxylic acid (TCA) cycle. The 
246 genome also contains a complete set of genes for an electron transport chain comprising Complexes I to

247 IV and an F-type ATPase. It has two terminal oxidases; a bd-type quinol and a cbb3-type cytochrome

248 (Complex IV), both of which are used for microaerobic respiration (Preisig et al., 1996). According to

249 PHX (predicted highly expressed) analysis (Karlin \& Mrázek, 2000), many of the genes in the glycolysis

250 pathway, TCA cycle and electron transport chain are predicted to be highly expressed (Fig. 2; Table S2)

251 suggesting oxidative metabolism is central to the predatory lifestyle of $V$. chlorellavorus despite the

252 inference of adaptation to low oxygen conditions. However, the genome also contains lactate

253 dehydrogenase suggesting that it is able to ferment pyruvate to lactate under anaerobic conditions (Fig.

254 2). The bacterium contains genes for fatty acid biosynthesis and $\beta$-oxidation, which leads to the

255 production of acetyl-CoA. Consistent with other described members of the class Melainabacteria, and in

256 contrast to oxygenic photosynthetic cyanobacteria, $V$. chlorellavorus lacks genes for photosynthesis and

257 carbon fixation (Soo et al., 2014). V. chlorellavorus can synthesise its own nucleotides and several

258 cofactors and vitamins including lipoate, nicotinate, heme, riboflavin and thiamine-diphosphate, but only

25915 amino acids: alanine, asparagine, aspartate, cysteine, glutamate, glutamine, glycine, isoleucine,

260 leucine, lysine, methionine, proline, threonine, tryptophan and valine. Although V. chlorellavorus does

261 not have the genes necessary to synthesise the remaining five amino acids or their polyamine

262 derivatives, it contains amino acid and polyamine transporters (Fig. 2) that would allow it to obtain these

263 organic compounds from external sources, most likely C. vulgaris.

264

265 The predatory lifestyle of Vampirovibrio chlorellavorus

266 Based on genomic inference and electron microscopy images obtained by Coder \& Starr (1978), we

267 divide the predatory life cycle of $V$. chlorellavorus into five phases comprising i) prey location, ii)

268 attachment, iii) formation of secretion apparatus and ingestion, iv) binary division and v) release (Fig.

$2693)$. 


\section{Phase i: Prey location}

272 The $V$. chlorellavorus genome encodes two-component regulatory systems including the well-known

273 CheA-CheY signal transduction pathway that couples to flagella rotation or pili extension, attachment

274 and retention (Fig. 2) allowing the cell to move towards chemoattractants or away from chemorepellents

275 (Wadhams \& Armitage, 2004). Coder and Starr (1978) showed that $V$. chlorellavorus is able to swim

276 towards its prey using a single, polar unsheathed flagellum possibly assisted by pili visible as thick

277 bundles in proximity to the flagellum. All of the genes necessary to produce a functional flagellum and

278 type IV pili (TFP) are present in the $V$. chlorellavorus genome ((Macnab 2003); Table S3). In

279 Cyanobacteria, Synechocystis strain PCC 6803 uses TFP for motility and it has also been speculated that

280 TFP can drive motility in Nostoc punctiforme (Bhaya et al., 2000; Duggan et al., 2007). It is likely that

$281 V$. chlorellavorus uses chemotaxis to help it locate prey, but based on genome inference alone, it is not 282 possible to determine which gradients $V$. chlorellavorus is detecting and responding to. However, the 283 genome does contain one globin-coupled sensor inferred to be used for aerotaxis ((Freitas et al., 2003);

284 Fig. S3) and one putative light-activated kinase (bacteriophytochrome; (Bhoo et al. 2001); BphP in Fig.

285 2) that may enable $V$. chlorellavorus to move towards oxic and illuminated regions of its habitat that

286 have a higher likelihood of containing Chlorella cells.

288 Phase ii: Attachment and formation of a conjugative secretion apparatus

$289 V$. chlorellavorus has a number of cellular features that likely facilitate its observed attachment to

290 Chlorella cells: TFP (described above), an outer membrane protein (OmpA) and von Willebrand

291 domain-containing proteins. While there are no reports of bacteria adhering to unicellular microbial

292 eukaryotes using these structures, there are a number of examples for adherence to animal tissues. TFP

293 are known to be involved in adhesion of pathogenic Escherichia coli and Neisseria meningitidis to 
294 human epithelial cells as a key virulence mechanism (Chamot-Rooke et al., 2011; Pizarro-Cerdá \&

295 Cossart, 2006). OmpA porins are outer membrane proteins that assemble into an eight stranded $\beta$-barrel

296 structure with four surface-exposed loops. Shin et al. (2005), showed that OmpA surface loops are

297 critical for adhesion of $E$. coli to brain microvascular endothelial cells leading to neonatal meningitis

298 (Shin et al., 2005). Furthermore, OmpA is involved in the binding of Acinetobacter baumanii and

299 Pasteurella multocida to fibronectin from human lung carcinoma (Smani et al., 2012). The von

300 Willebrand factor A (VWA) domains are found predominantly in cell adhesion and extracellular matrix

301 molecules, including integrins, hemicentins and matrilins (Whittaker \& Hynes, 2002). Enterococcus

302 faecalis VWA domains are able to mediate protein-protein adhesion through a metal ion-dependent

303 adhesion site (Nielsen et al., 2012).

304

305 Ultrastructural studies have shown that $V$. chlorellavorus forms a discrete pad of unknown composition

306 during attachment to Chlorella cells (Gromov \& Mamkaeva, 1972; Mamkaeva \& Rybal'chenko, 1979).

307 Similar pads are involved in the attachment of the uncultured predatory bacterium Vampirococcus to its

308 bacterial prey, Chromatium (Guerrero et al., 1986). Spikes of electron dense material have been

309 observed to extend from the $V$. chlorellavorus pad into the Chlorella cell through the algal cell envelope

310 (Coder \& Starr, 1978). We propose that the attachment pad and spike are a type IV secretion system

311 (T4SS) fully encoded in the $V$. chlorellavorus genome in three operons (Fig. 2 and Fig. S4).

312 Phylogenetic analysis of the VirB4 ATPase (gene trbE), a highly conserved component of the T4SS

313 used to classify these secretion systems (Guglielmini et al., 2013) showed that the $V$. chlorellavorus

314 orthologue is most closely related to a T-type conjugation system in Nitrosomonas eutropha (Fig. S5).

315 T-type conjugation T4SS are best known in Agrobacterium tumefaciens which form a secretion channel

316 through which the T-strand (the strand destined for transfer) is passed into plant cells causing crown gall

317 disease (Christie, 2004). More generally, T-type conjugation systems can pass single stranded DNA and 
318 proteins into recipient cells (Alvarez-Martinez \& Christie, 2009). Two of the T4SS operons of $V$.

319 chlorellavorus are found on conjugative plasmids (Fig. S4), which are predicted to be made

320 singlestranded by their relaxases, nicking the DNA at the origin of transfer and transporting the T-strand

321 to the Chlorella cell via the conjugation channel. The T-strand would then integrate into the Chlorella

322 chromosome and be expressed (Cascales \& Christie, 2003) (Fig. 4). Since the nature of the relationship

323 between the two conjugating cells is predatory, we may expect that the T-strand would carry genes that

324 facilitate ingestion of the Chlorella cell contents. No genes encoding hydrolytic enzymes were identified 325 on the plasmids, though one encodes several efflux transporters (Fig. S4; see below).

\section{Phase iii: Ingestion}

328 Five to seven days after $V$. chlorellavorus attachment, Chlorella cells remain intact but are devoid of

329 cytoplasmic contents and contain only large vacuolated areas and membranous structures which are

330 presumed to be organellar remains (Coder \& Starr, 1978). The V. chlorellavorus genome encodes

331 numerous proteins that may be involved in the observed ingestion of Chlorella cell contents, including

332108 proteases and 123 carbohydrate-active enzymes (Tables S4 and S5). The majority of the latter

333 group are glycoside hydrolases which are predicted to degrade polysaccharides and glycoproteins, major

334 components of the Chlorella cell envelope (Gerken et al., 2013) as well as starch and glycogen, which

335 are diurnally stored as energy sources in Chlorella (Nakamura \& Miyachi, 1982). Extracellular proteases

336 are produced by many bacterial pathogens and are commonly involved in the degradation of the host

337 extracellular matrix, facilitating invasion and colonisation (Kennan et al., 2010). They have also been

338 suggested as important factors in virulence for other predatory bacteria, for example Bdellovibrio

339 bacteriovorus and Micavibrio aeruginosa (Rendulic et al., 2004; Wang et al., 2011). The $V$.

340 chlorellavorus genome contains an alginate lyase, an enzyme that is able to degrade alginate via $\beta$ -

341 elimination cleavage of glycosidic bonds in the polysaccharide backbone (Lamppa et al., 2011). Alginate 


\section{PeerJ Reviewing Manuscript}

342 is a common component of marine brown algae cell envelopes and intracellular material which is

343 targeted as a carbon and energy source by bacteria possessing alginate lyases (Wong et al., 2000).

344 Chlorella cells may similarly contain alginate supported by the finding of an alginate lyase gene in a

345 Chlorella virus (Suda et al., 1999). We propose that this suite of hydrolytic enzymes are synthesised in

$346 V$. chlorellavorus and transported via the T4SS conjugation channel into the prey cell where they

347 produce hydrolysates in the Chlorella cell (Fig. 3). The T4SS plasmid-encoded efflux transporters (Fig.

348 S4) may facilitate the export of lysates from the Chlorella cell assuming that the T-strand is integrated

349 and expressed in Chlorella as is the case in Agrobacterium tumour formation (Christie, 2004). Lysates

350 exported into the surrounding milieu could then be imported into the attached $V$. chlorellavorus cell (and

351 possibly neighbouring predatory cells) using a number of transport systems from the ATP-binding

352 cassette (ABC) superfamily, the Major Facilitator Superfamily and/or permeases encoded in the bacterial

353 genome (Fig. 2). It is unlikely that Chlorella lysates would be directly transported into $V$. chlorellavorus

354 cells via the conjugation channel as conjugation systems have only been shown to deliver protein or

355 DNA substrates to eukaryotic target cells but not vice versa (Cascales \& Christie, 2003).

357 Phase iv: Binary fission

358 Attached $V$. chlorellavorus cells have been observed to divide by binary fission presumably using

359 nutrients and energy derived from ingestion of Chlorella lysates, consistent with an obligate predatory

360 lifestyle (Coder \& Starr, 1978; Gromov \& Mamkaeva, 1980). The genome contains the cell division

361 proteins required to replicate by this process, including the tubulin-like protein FtsZ, which is predicted

362 to be highly expressed by PHX analysis, and the regulation of the placement of division site genes,

$363 \min C,-D$ and $-E$ (Lutkenhaus \& Addinall, 1997). 
366 A new lifecycle is started when progeny cells release from consumed Chlorella cells (Fig. 3). Released

367 cells then synthesise flagella to aid their dispersal and have a range of mechanisms to protect themselves

368 from environmental stress as free-living organisms. The $V$. chlorellavorus genome encodes two

369 superoxide dismutases, which convert $\mathrm{O}_{2}{ }^{-}$to $\mathrm{H}_{2} \mathrm{O}_{2}$ and $\mathrm{O}_{2}$ (Cabiscol et al., 2000) and one catalase-

370 peroxidase, $k a t G$, a $\mathrm{H}_{2} \mathrm{O}_{2}$ scavenger (Jittawuttipoka et al., 2009). Both of these enzymes can be used to

371 combat oxidative stress that may be induced by environmental agents such as radiation or compounds

372 that can generate intracellular $\mathrm{O}_{2}^{-}$(Cabiscol et al., 2000) or from the Chlorella (Mallick \& Mohn, 2000).

373 The genome encodes a large and small conductance mechanosensitive channel protein that prevents cells

374 from lysing upon sudden hypo-osmotic shock by releasing solutes and water (Birkner et al., 2012). It

375 also encodes a protein containing a stress-induced bacterial acidophilic repeat motif and three copies of a

376 universal stress protein (UspA), an autophosphorylating serine and threonine phosphoprotein (Kvint et

377 al. 2003). In other stress conditions, such as temperature shock, starvation or the presence of oxidants or

378 DNA-damaging agents, the expression of UspA is increased or decreased, which is known to be

379 correlated with improved bacterial survival (Jenkins et al., 2011). Beta-lactamases, cation/multidrug

380 efflux pumps and ABC-type multidrug and solvent transport systems were identified (Fig. 2) that could

381 be used to eliminate antibiotics or toxins encountered in the environment (Frère, 1995; Lubelski et al.,

382 2007).

383

384 Comparison of $\boldsymbol{V}$. chlorellavorus to other predatory bacteria

385 Pasternak et al. 2013, conducted a study of 11 predatory and 19 non-predatory bacterial genomes to

386 define the 'predatome', the core gene set proposed for bacteria with predatory lifestyles (Pasternak et al.,

387 2013). The study found that the most striking difference between predators and non-predators is their

388 method of synthesising isoprenoids. All predators, except for M. aeruginosavorus, encode the three

389 essential enzymes used in the mevalonate pathway, which is uncommon in bacteria, whereas non- 
390 predators encode five essential enzymes for the more typical non-mevalonate pathway. It was suggested

391 that predatory bacteria may have access to acetoacetyl-CoA pools in their prey cells, which is the first

392 substrate used in the mevalonate pathway (Pasternak et al., 2013). However, V. chlorellavorus lacks two

393 of the three mevalonate pathway genes and instead encodes the non-mevalonate pathway (Fig. 2).

394 Twelve additional protein families were identified as specific to the predator set including those

395 involved in chemotaxis, cell adhesion, degradation of polypeptides and benzoate, and four enzymes that

396 may have evolved to scavenge essential metabolites (Pasternak et al., 2013). V. chlorellavorus has

397 orthologues of eight of these protein families and while lacking some of the specific adhesion and

398 degradation genes (OrthoMCL OG4 39191, 26993, 21243, 18254), it encodes alternative proteins for

399 these functions (see above). Eleven additional protein families were identified as specific to the non-

400 predatory bacteria including those for riboflavin and amino acid synthesis, specifically tryptophan,

401 phenylalanine, tyrosine, valine, leucine and isoleucine (Pasternak et al., 2013). V. chlorellavorus has all

402 but one of these "non-predatory" genes (OrthoMCL OG4 11203) which may reflect its phylogenetic

403 novelty given that the core set analysis was based mostly on comparison of Proteobacteria (Pasternak et

404 al., 2013). We note that while V. chlorellavorus can make these particular compounds, its cofactor and

405 amino acid biosynthesis repertoire is limited (5 cofactors, 15 amino acids).

406

407 Comparison of $\boldsymbol{V}$. chlorellavorus to other Melainabacteria genomes

408 Consistent with all sequenced representatives of the class Melainabacteria (Di Rienzi et al., 2013; Soo et

409 al., 2014), $V$. chlorellavorus is missing all recognised photosynthesis genes including those for

410 Photosystems I and II, chlorophyll and antennae proteins. This supports the hypothesis that

411 photosynthetic cyanobacteria acquired photosystems after diverging from the ancestor of the

412 Melainabacteria ((Di Rienzi et al., 2013; Soo et al., 2014); Fig. 1). The $V$. chlorellavorus genome falls

413 within the size range of previously reported Melainabacteria (1.8 to $5.5 \mathrm{Mbp}$ ) but has the highest GC 
414 content thus far (51.4\%) compared with the GC content of other Melainabacteria who have a range of

$41527.5 \%$ to $49.4 \%$. V. chlorellavorus is the second representative of the class inferred to be capable of

416 oxidative phosphorylation as it contains a full respiratory chain (Fig. 2), the other being Obscuribacter

417 phosphatis (Soo et al., 2014). V. chlorellavorus encodes a flagellum which is also found in some

418 representatives of the order Gastranaerophilales (ACD20, MEL_B1 and MEL_B2). We inferred a

419 phylogenetic tree for the conserved flagella marker gene, fliI (Minamino \& Namba 2008) and found that

420 the Melainabacteria fli genes form a monophyletic cluster consistent with their internal branching order

421 in the genome tree (Fig. 1 and Fig. S6) This association suggests that flagella were present in the

422 cyanobacterial ancestor of the Gastranaerophilales and Vampirovibrionales and were subsequently lost

423 at least once in the Gastranaerophilales (Fig. 1). A global comparison of COG (clusters of orthologous

424 groups) categories revealed that $V$. chlorellavorus has a functional distribution typical of other

425 Melainabacteria genomes with the exception of genes involved in intracellular trafficking, secretion, and

426 vesicular transport (Fig. S7). V. chlorellavorus is overrepresented in this category due to a higher

427 proportion of genes involved in Type IV secretion systems, which we posit to be important in the

428 lifecycle of this predator (see above).

\section{Conclusions}

431 We have sequenced and assembled a near complete genome from a 36-year old lyophilised co-culture of

432 the predatory bacterium Vampirovibrio chlorellavorus. Comparative gene and genome analyses confirm

433 that $V$. chlorellavorus is a member of the Melainabacteria, a recently described non-photosynthetic class

434 in the cyanobacterial phylum (Soo et al., 2014). V. chlorellavorus is the first recognised member of the

435 Cyanobacteria with a predatory lifecycle and we predict that it is the first predator to use a conjugative

436 type IV secretion system similar to Agrobacterium tumefaciens to invade its host. It remains to be 
437 determined how widespread this phenotype is within the Melainabacteria and how it may have evolved 438 from non-predatory cyanobacterial ancestors.

\section{Acknowledgements}

441 We thank Jim Prosser, Samantha Law and Tina Niven from NCIMB for their help with obtaining the co442 cultures of $V$. chlorellavorus and $C$. vulgaris and Serene Lowe for preparing the DNA for sequencing 443 and IMB, UQ for sequencing. We also thank Xuyen Le and Bryan Wee for discussions on motility and 444 T4SS, Julien Guglielmini for data on T4SS, Rick Webb for inspection of S-layers in transmission 445 electron microscopy images, Michael Nefedov for translation of Russian manuscripts and Nancy 446 Lachner for attempts to extract RNA from the lyophilised cells. 


\section{References}

450 Altschul SF, Gish W, Miller W, Myers EW, and Lipman DJ. 1990. Basic local alignment search tool. $J$ 451 Mol Biol 215:403-410.

452 Alvarez-Martinez CE, and Christie PJ. 2009. Biological diversity of prokaryotic type IV secretion 453 systems. Microbiol Mol Biol Rev 73:775-808.

454 Andrews S. 2010. FastQC: A quality control tool for high throughput sequence data. Reference Source.

455 Beveridge TJ. 1999. Structures of Gram-Negative Cell Walls and Their Derived Membrane Vesicles. $J$ 456 Bacteriol 181:4725-4733.

Bhaya D, Bianco NR, Bryant D, and Grossman A. 2000. Type IV pilus biogenesis and motility in the 458 cyanobacterium Synechocystis sp. PCC6803. Molecular Microbiology 37:941-951.

Bhoo S-H, Davis SJ, Walker J, Karniol B, and Vierstra RD. 2001. Bacteriophytochromes are photochromic histidine kinases using a biliverdin chromophore. Nature 414:776-779.

461

462

463

464

Birkner JP, Poolman B, and Koçer A. 2012. Hydrophobic gating of mechanosensitive channel of large conductance evidenced by single-subunit resolution. Proceedings of the National Academy of Sciences 109:12944-12949.

Cabiscol E, Tamarit J, and Ros J. 2000. Oxidative stress in bacteria and protein damage by reactive oxygen species. International Microbiology 3:3-8.

Cascales E, and Christie PJ. 2003. The versatile bacterial type IV secretion systems. Nat Rev Micro 1:137-149.

Casida LE. 1983. Interaction of Agromyces ramosus with Other Bacteria in Soil. Applied and Environmental Microbiology 46:881-888.

Chamot-Rooke J, Mikaty G, Malosse C, Soyer M, Dumont A, Gault J, Imhaus A-F, Martin P, Trellet M, Clary G, Chafey P, Camoin L, Nilges M, Nassif X, and Duménil G. 2011. Posttranslational Modification of Pili upon Cell Contact Triggers N. meningitidis Dissemination. Science 331:778-782.

Chaudhuri RR, Loman NJ, Snyder LAS, Bailey CM, Stekel DJ, and Pallen MJ. 2008. xBASE2: a comprehensive resource for comparative bacterial genomics. Nucleic Acids Research 36:D543D546.

Chen F, Mackey AJ, Stoeckert CJ, and Roos DS. 2006. OrthoMCL-DB: querying a comprehensive multi-species collection of ortholog groups. Nucleic Acids Research 34:D363-D368. 
479 Christie PJ. 2004. Type IV secretion: the Agrobacterium VirB/D4 and related conjugation systems. Biochimica et Biophysica Acta (BBA) - Molecular Cell Research 1694:219-234.

481 Coder D, and Starr M. 1978. Antagonistic association of the chlorellavorus bacterium ("Bdellovibrio" 482 chlorellavorus) with Chlorella vulgaris. Current Microbiology 1:59-64.

483 Coder DM, and Goff LJ. 1986. The host range of the Chlorellavorous bacterium ("Vampirovibrio 484 chlorellavorus"). Journal of Phycology 22:543-546.

485 Corbett LL, and Parker DL. 1976. Viability of lyophilized cyanobacteria (blue-gree algae). Applied and 486 Environmental Microbiology 32:777-780.

Di Rienzi SC, Sharon I, Wrighton KC, Koren O, Hug LA, Thomas BC, Goodrich JK, Bell JT, Spector 488 TD, Banfield JF and Ley RE. 2013. The human gut and groundwater harbor non-photosynthetic bacteria belonging to a new candidate phylum sibling to Cyanobacteria. eLife 2:e01102.

Divakaruni AV, Baida C, White CL, and Gober JW. 2007. The cell shape proteins MreB and MreC control cell morphogenesis by positioning cell wall synthetic complexes. Molecular Microbiology 66:174-188.

Duggan PS, Gottardello P, and Adams DG. 2007. Molecular Analysis of Genes in Nostoc punctiforme Involved in Pilus Biogenesis and Plant Infection. J Bacteriol 189:4547-4551.

Felsenstein J. 1985. Confidence Limits on Phylogenies: An Approach Using the Bootstrap. Evolution 39:783-791.

Felsenstein J. 1989. PHYLIP - Phylogeny inference package (Version 3.2). Cladistics 5:164-166.

Finn RD, Bateman A, Clements J, Coggill P, Eberhardt RY, Eddy SR, Heger A, Hetherington K, Holm L, Mistry J, Sonnhammer EL, Tate J, and Punta M. 2014. Pfam: the protein families database. Nucleic Acids Res 42:D222-230.

Freitas TAK, Hou S, and Alam M. 2003. The diversity of globin-coupled sensors. FEBS Letters 552:99-104.

Frère J-M. 1995. Beta-lactamases and bacterial resistance to antibiotics. Molecular Microbiology 16:385-395.

Gerken HG, Donohoe B, and Knoshaug EP. 2013. Enzymatic cell wall degradation of Chlorella vulgaris and other microalgae for biofuels production. Planta 237:239-253.

Gromov BV, and Mamkaeva KA. 1972. Electron microscopic study of parasitism by Bdellovibrio chlorellavorus bacteria on cells of the green alga Chlorella vulgaris. Tsitologiia 14:256-260. 
509 Gromov BV, and Mamkaeva KA. 1980. New genus of bacteria, Vampirovibrio, parasitizing chlorella and previously assigned to the genus Bdellovibrio. Mikrobiologiia 49:165-167.

511 Guerrero R, Pedrós-Alió C, Esteve I, Mas J, Chase D, and Margulis L. 1986. Predatory prokaryotes:

512 Predation and primary consumption evolved in bacteria. Proceedings of the National Academy 513 of Sciences 83:2138-2142.

514 Guglielmini J, de la Cruz F, and Rocha EP. 2013. Evolution of conjugation and type IV secretion $515 \quad$ systems. Mol Biol Evol 30:315-331.

516 Guindon S, Dufayard J-F, Lefort V, Anisimova M, Hordijk W, and Gascuel O. 2010. New Algorithms and Methods to Estimate Maximum-Likelihood Phylogenies: Assessing the Performance of PhyML 3.0. Systematic Biology 59:307-321.

Haft DH, Selengut JD, and White O. 2003. The TIGRFAMs database of protein families. Nucleic Acids Res 31:371-373.

Hyatt D, Chen G-L, LoCascio P, Land M, Larimer F, and Hauser L. 2010. Prodigal: prokaryotic gene recognition and translation initiation site identification. BMC Bioinformatics 11:119.

Hyatt D, LoCascio PF, Hauser LJ, and Uberbacher EC. 2012. Gene and translation initiation site prediction in metagenomic sequences. Bioinformatics 28:2223-2230.

Jenkins R, Burton N, and Cooper R. 2011. Effect of manuka honey on the expression of universal stress protein A in meticillin-resistant Staphylococcus aureus. International Journal of Antimicrobial Agents 37:373-376.

Jittawuttipoka T, Buranajitpakorn S, Vattanaviboon P, and Mongkolsuk S. 2009. The CatalasePeroxidase KatG Is Required for Virulence of Xanthomonas campestris pv. campestris in a Host Plant by Providing Protection against Low Levels of H2O2. J Bacteriol 191:7372-7377.

Jones P, Binns D, Chang H-Y, Fraser M, Li W, McAnulla C, McWilliam H, Maslen J, Mitchell A, Nuka G, Pesseat S, Quinn AF, Sangrador-Vegas A, Scheremetjew M, Yong S-Y, Lopez R, and Hunter S. 2014. InterProScan 5: genome-scale protein function classification. Bioinformatics (Oxford, England) 30:1236-1240.

Jurkevitch E. 2007. Predatory behaviors in bacteria-diversity and transitions. Microbe-american society for microbiology 2:67.

Kanehisa M, Goto S, Kawashima S, Okuno Y, and Hattori M. 2004. The KEGG resource for deciphering the genome. Nucleic Acids Research 32:D277-D280. 
Karlin S, and Mrázek J. 2000. Predicted Highly Expressed Genes of Diverse Prokaryotic Genomes. $J$ Bacteriol 182:5238-5250.

Kennan RM, Wong W, Dhungyel OP, Han X, Wong D, Parker D, Rosado CJ, Law RHP, McGowan S, Reeve SB, Levina V, Powers GA, Pike RN, Bottomley SP, Smith AI, Marsh I, Whittington RJ, Whisstock JC, Porter CJ, and Rood JI. 2010. The Subtilisin-Like Protease AprV2 Is Required for Virulence and Uses a Novel Disulphide-Tethered Exosite to Bind Substrates. PLoS Pathog 6:e1001210.

Kiss H, Nett M, Domin N, Martin K, Maresca JA, Copeland A, Lapidus A, Lucas S, Berry KW, Glavina Del Rio T, Dalin E, Tice H, Pitluck S, Richardson P, Bruce D, Goodwin L, Han C, Detter JC, Schmutz J, Brettin T, Land M, Hauser L, Kyrpides NC, Ivanova N, Goker M, Woyke T, Klenk HP, and Bryant DA. 2011. Complete genome sequence of the filamentous gliding predatory bacterium Herpetosiphon aurantiacus type strain (114-95(T)). Stand Genomic Sci 5:356-370.

Kvint K, Nachin L, Diez A, and Nyström T. 2003. The bacterial universal stress protein: function and regulation. Current Opinion in Microbiology 6:140-145.

Lamppa JW, Ackerman ME, Lai JI, Scanlon TC, and Griswold KE. 2011. Genetically Engineered Alginate Lyase-PEG Conjugates Exhibit Enhanced Catalytic Function and Reduced Immunoreactivity. PLoS ONE 6:e17042.

Langille MG, and Brinkman FS. 2009. IslandViewer: an integrated interface for computational identification and visualization of genomic islands. Bioinformatics 25:664-665.

Lubelski J, Konings WN, and Driessen AJM. 2007. Distribution and Physiology of ABC-Type Transporters Contributing to Multidrug Resistance in Bacteria. Microbiology and Molecular Biology Reviews 71:463-476.

Ludwig W, Strunk O, Westram R, Richter L, Meier H, Yadhukumar BA, Buchner A, Lai T, Steppi S, Jobb G, Förster W, Brettske I, Gerber S, Ginhart AW, Gross O, Grumann S, Hermann S, Jost R, König A, Liss T, Lüssmann R, May M, Nonhoff B, Reichel B, Strehlow R, Stamatakis A, Stuckmann N, Vilbig A, Lenke M, Ludwig T, Bode A, Schleifer KH. 2004. ARB: a software environment for sequence data. Nucleic Acids Research 32:1363-1371.

Lutkenhaus J, and Addinall SG. 1997. Bacterial cell division and the Z ring. Annu Rev Biochem 66:93116.

Macnab RM. 2003. How bacteria assemble flagella. Annu Rev Microbiol 57:77-100. 
570 Mallick N, and Mohn FH. 2000. Reactive oxygen species: response of algal cells. Journal of Plant Physiology 157:183-193.

Mamkaeva KA, and Rybal'chenko OV. 1979. Ultrastructural characteristics of Bdellovibrio chlorellavorus. Mikrobiologiia 48:159-161.

Markowitz VM, Chen IMA, Chu K, Szeto E, Palaniappan K, Pillay M, Ratner A, Huang J, Pagani I, Tringe S, Huntemann M, Billis K, Varghese N, Tennessen K, Mavromatis K, Pati A, Ivanova NN, and Kyrpides NC. 2014. IMG/M 4 version of the integrated metagenome comparative analysis system. Nucleic Acids Research 42:D568-D573.

Markowitz VM, Mavromatis K, Ivanova NN, Chen IMA, Chu K, and Kyrpides NC. 2009. IMG ER: a system for microbial genome annotation expert review and curation. Bioinformatics 25:22712278.

McDonald D, Price MN, Goodrich J, Nawrocki EP, DeSantis TZ, Probst A, Andersen GL, Knight R, and Hugenholtz P. 2012. An improved Greengenes taxonomy with explicit ranks for ecological and evolutionary analyses of bacteria and archaea. ISME J 6:610-618.

Minamino T, and Namba K. 2008. Distinct roles of the FliI ATPase and proton motive force in bacterial flagellar protein export. Nature 451:485-488.

Nakamura Y, and Miyachi S. 1982. Effect of Temperature on Starch Degradation in Chlorella vulgaris 11h Cells. Plant and Cell Physiology 23:333-341.

Nielsen HV, Guiton PS, Kline KA, Port GC, Pinkner JS, Neiers F, Normark S, Henriques-Normark B, Caparon MG, and Hultgren SJ. 2012. The Metal Ion-Dependent Adhesion Site Motif of the Enterococcus faecalis EbpA Pilin Mediates Pilus Function in Catheter-Associated Urinary Tract Infection. mBio 3.

Parks DH, Imelfort M, Skennerton CT, Hugenholtz P, and Tyson GW. 2014a. CheckM: assessing the quality of microbial genomes recovered from isolates, single cells, and metagenomes. PeerJ PrePrints 2:e554v551.

Parks DH, Tyson GW, Hugenholtz P, and Beiko RG. 2014b. STAMP: statistical analysis of taxonomic and functional profiles. Bioinformatics 30:3123-3124.

Pasternak Z, Pietrokovski S, Rotem O, Gophna U, Lurie-Weinberger MN, and Jurkevitch E. 2013. By their genes ye shall know them: genomic signatures of predatory bacteria. ISME J 7:756-769.

Pizarro-Cerdá J, and Cossart P. 2006. Bacterial Adhesion and Entry into Host Cells. Cell 124:715-727. 
600 Preisig O, Zufferey R, Thöny-Meyer L, Appleby CA, and Hennecke H. 1996. A high-affinity cbb3601

602 type cytochrome oxidase terminates the symbiosis-specific respiratory chain of Bradyrhizobium japonicum. Journal of Bacteriology 178:1532-1538.

Price MN, Dehal PS, and Arkin AP. 2009. FastTree: Computing Large Minimum Evolution Trees with Profiles instead of a Distance Matrix. Molecular Biology and Evolution 26:1641-1650.

Quail MA, Swerdlow H, and Turner DJ. 2009. Improved protocols for the illumina genome analyzer sequencing system. Curr Protoc Hum Genet Chapter 18:Unit 18.12.

Quast C, Pruesse E, Yilmaz P, Gerken J, Schweer T, Yarza P, Peplies J, and Glöckner FO. 2013. The SILVA ribosomal RNA gene database project: improved data processing and web-based tools. Nucleic Acids Research 41:D590-D596.

Rawlings ND, Waller M, Barrett AJ, and Bateman A. 2014. MEROPS: the database of proteolytic enzymes, their substrates and inhibitors. Nucleic Acids Research 42:D503-D509.

Rendulic S, Jagtap P, Rosinus A, Eppinger M, Baar C, Lanz C, Keller H, Lambert C, Evans KJ, Goesmann A, Meyer F, Sockett RE, and Schuster SC. 2004. A Predator Unmasked: Life Cycle of Bdellovibrio bacteriovorus from a Genomic Perspective. Science 303:689-692.

Sára M, and Sleytr UB. 2000. S-Layer Proteins. J Bacteriol 182:859-868.

Saw JH, Yuryev A, Kanbe M, Hou S, Young AG, Aizawa S, and Alam M. 2012. Complete genome sequencing and analysis of Saprospira grandis str. Lewin, a predatory marine bacterium. Stand Genomic Sci 6:84-93.

Seemann T. 2014. Prokka: rapid prokaryotic genome annotation. Bioinformatics.

Shin S, Lu G, Cai M, and Kim KS. 2005. Escherichia coli outer membrane protein A adheres to human brain microvascular endothelial cells. Biochem Biophys Res Commun 330:1199-1204.

Smani Y, McConnell MJ, and Pachón J. 2012. Role of Fibronectin in the Adhesion of Acinetobacter baumanniito Host Cells. PLoS ONE 7:e33073.

Šmarda J, Šmajs D, Komrska J, and Krzyžánek V. 2002. S-layers on cell walls of cyanobacteria. Micron 33:257-277.

Soo RM, Skennerton CT, Sekiguchi Y, Imelfort M, Paech SJ, Dennis PG, Steen JA, Parks DH, Tyson GW, and Hugenholtz P. 2014. An Expanded Genomic Representation of the Phylum Cyanobacteria. Genome Biology and Evolution. 6:1031-1045.

Stamatakis A. 2006. RAxML-VI-HPC: maximum likelihood-based phylogenetic analyses with thousands of taxa and mixed models. Bioinformatics 22:2688-2690. 
631 Stolp H, and Starr MP. 1963. Bdellovibrio bacteriovorus gen. et sp. n., a predatory, ectoparasitic, and bacteriolytic microorganism. Antonie Van Leeuwenhoek 29:217-248.

633 Suda K, Tanji Y, Hori K, and Unno H. 1999. Evidence for a novel Chlorella virus-encoded alginate lyase. FEMS Microbiology Letters 180:45-53.

Suzek BE, Huang H, McGarvey P, Mazumder R, and Wu CH. 2007. UniRef: comprehensive and nonredundant UniProt reference clusters. Bioinformatics 23:1282-1288.

Swofford DL, and Sullivan J. 2003. Phylogeny inference based on parsimony and other methods using PAUP*. The Phylogenetic Handbook: A Practical Approach to DNA and Protein Phylogeny 7:160-206.

Tatusov RL, Fedorova ND, Jackson JD, Jacobs AR, Kiryutin B, Koonin EV, Krylov DM, Mazumder R, Mekhedov SL, Nikolskaya AN, Rao BS, Smirnov S, Sverdlov AV, Vasudevan S, Wolf YI, Yin JJ, and Natale DA. 2003. The COG database: an updated version includes eukaryotes. BMC Bioinformatics 4:41.

The UniProt Consortium. 2008. The Universal Protein Resource (UniProt). Nucleic Acids Research 36:D190-D195.

Varma A, and Young KD. 2009. In Escherichia coli, MreB and FtsZ Direct the Synthesis of Lateral Cell Wall via Independent Pathways That Require PBP 2. J Bacteriol 191:3526-3533.

Velicer GJ, Kroos L, and Lenski RE. 2000. Developmental cheating in the social bacterium Myxococcus xanthus. Nature 404:598-601.

Waack S, Keller O, Asper R, Brodag T, Damm C, Fricke W, Surovcik K, Meinicke P, and Merkl R. 2006. Score-based prediction of genomic islands in prokaryotic genomes using hidden Markov models. BMC Bioinformatics 7:142.

Wadhams GH, and Armitage JP. 2004. Making sense of it all: bacterial chemotaxis. Nat Rev Mol Cell Biol 5:1024-1037.

Wang Z, Kadouri D, and Wu M. 2011. Genomic insights into an obligate epibiotic bacterial predator: Micavibrio aeruginosavorus ARL-13. BMC Genomics 12:453.

Whittaker CA, and Hynes RO. 2002. Distribution and Evolution of von Willebrand/Integrin A Domains: Widely Dispersed Domains with Roles in Cell Adhesion and Elsewhere. Molecular Biology of the Cell 13:3369-3387.

Wickham H. 2009. ggplot2: elegant graphics for data analysis: Springer. 
661 Wong TY, Preston LA, and Schiller NL. 2000. ALGINATE LYASE: Review of Major Sources and 662 Enzyme Characteristics, Structure-Function Analysis, Biological Roles, and Applications. Annu 663 Rev Microbiol 54:289-340.

664 Yin Y, Mao X, Yang J, Chen X, Mao F, and Xu Y. 2012. dbCAN: a web resource for automated 665 carbohydrate-active enzyme annotation. Nucleic Acids Res 40:W445-451.

666 


\section{Figure 1 (on next page)}

Phylogenetic position of Vampirovibrio chlorellavorus in the phylum Cyanobacteria

A) A maximum likelihood (ML) phylogenetic tree of the phylum Cyanobacteria inferred from a concatenated alignment of 109 single copy marker genes conserved across the bacterial domain. Black circles represent branch nodes with $>90 \%$ bootstrap support by ML analysis. Class Oxyphotobacteria group names are according to Shih et al. 2013. The blue and red arrow indicate putative acquisition and loss of flagella respectively in the class Melainabacteria. Representatives of 32 bacterial phyla were used as outgroups in the analysis (Fig. S2). $\mathrm{Ca}=$ Candidatus. B) A ML tree of the order Vampirovibrionales (Soo et al., 2014) based on aligned 16S rRNA gene sequences from the May, 2013 Greengenes database (McDonald et al., 2012). Black circles represent nodes with $>90 \% \mathrm{ML}$, maximum parsimony (MP) and neighbour joining (NJ) bootstrap support values. 


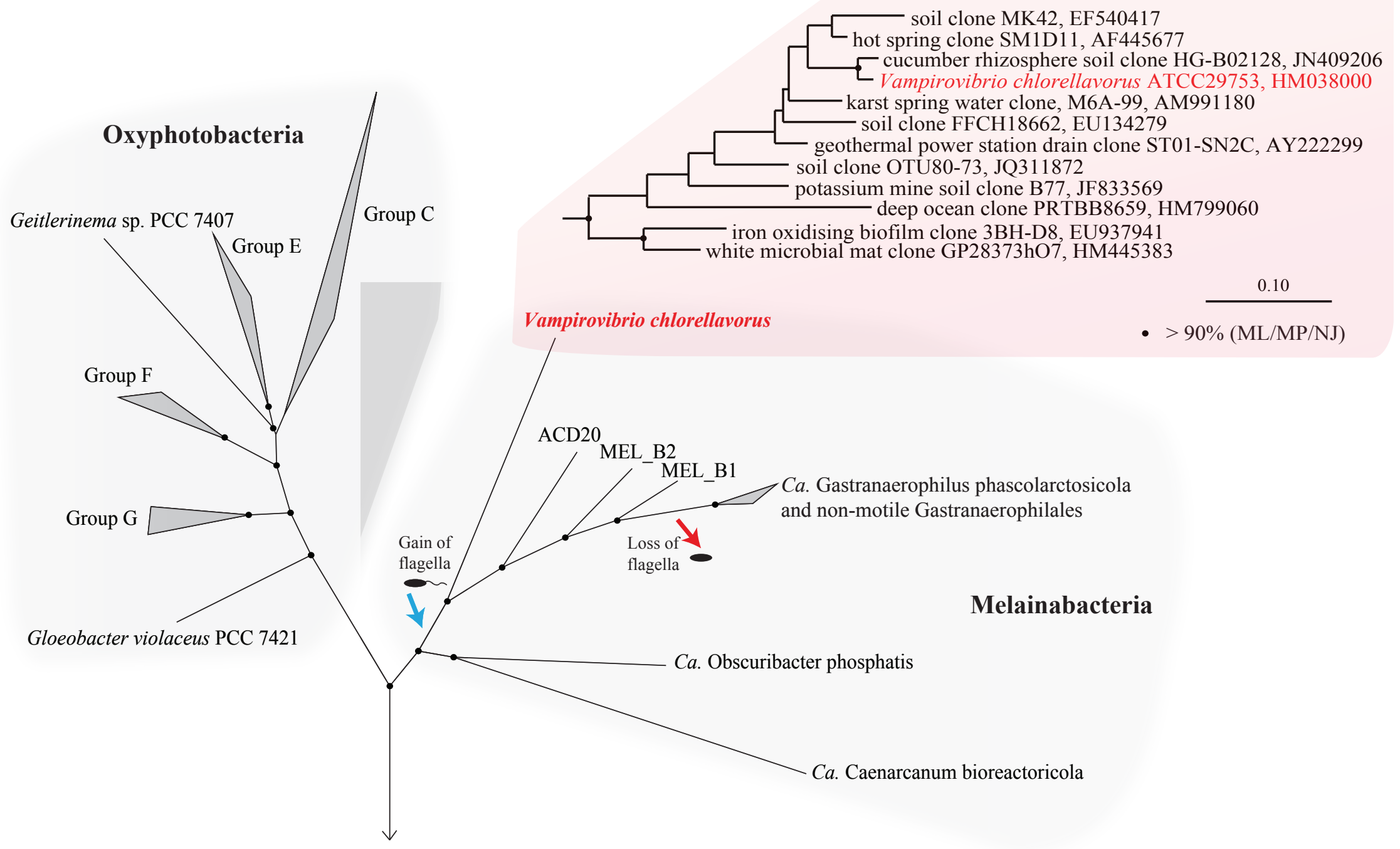




\section{Figure 2 (on next page)}

Metabolic reconstruction of Vampirovibrio chlorellavorus

Metabolic predictions for V. chlorellavorus based on genes annotated by IMG/ER (Markowitz et al., 2009). Soild and dashed lines represent single or multiple steps in a pathway respectively. Black ovals indicate substrates that enter the glycolysis pathway. Fermentation end-products are indicated as black rectangles. V. chlorellavorus is capable of oxidative phosphorylation as it contains a complete TCA cycle and electron transport chain.

Biosynthetic products are shown in green (amino acids), red (co-factors and vitamins), purple (nucleotides), and orange (non-mevalonate pathway products). Serine (highlighted in blue) is not able to be synthesised and is presumably transported into the cell. ATP-binding cassette transporters are highlighted in yellow and permeases, pumps and transporters are highlighted in orange. The direction of substrate transport across the membrane is shown with arrows. Putatively highly expressed genes and complexes are bolded. V. chlorellavorus is missing all recognised photosynthesis genes including those for Photosystems I and II, chlorophyll and antennae proteins. 
Potassium/proton Cation Peer: Reviewing Manuscript

Potassium/proton eftux
antiporter CPA2

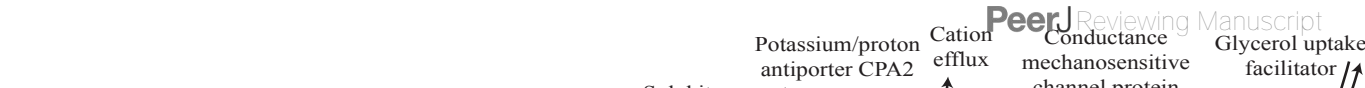
Sulphite exposphate/sulphate
Phosp

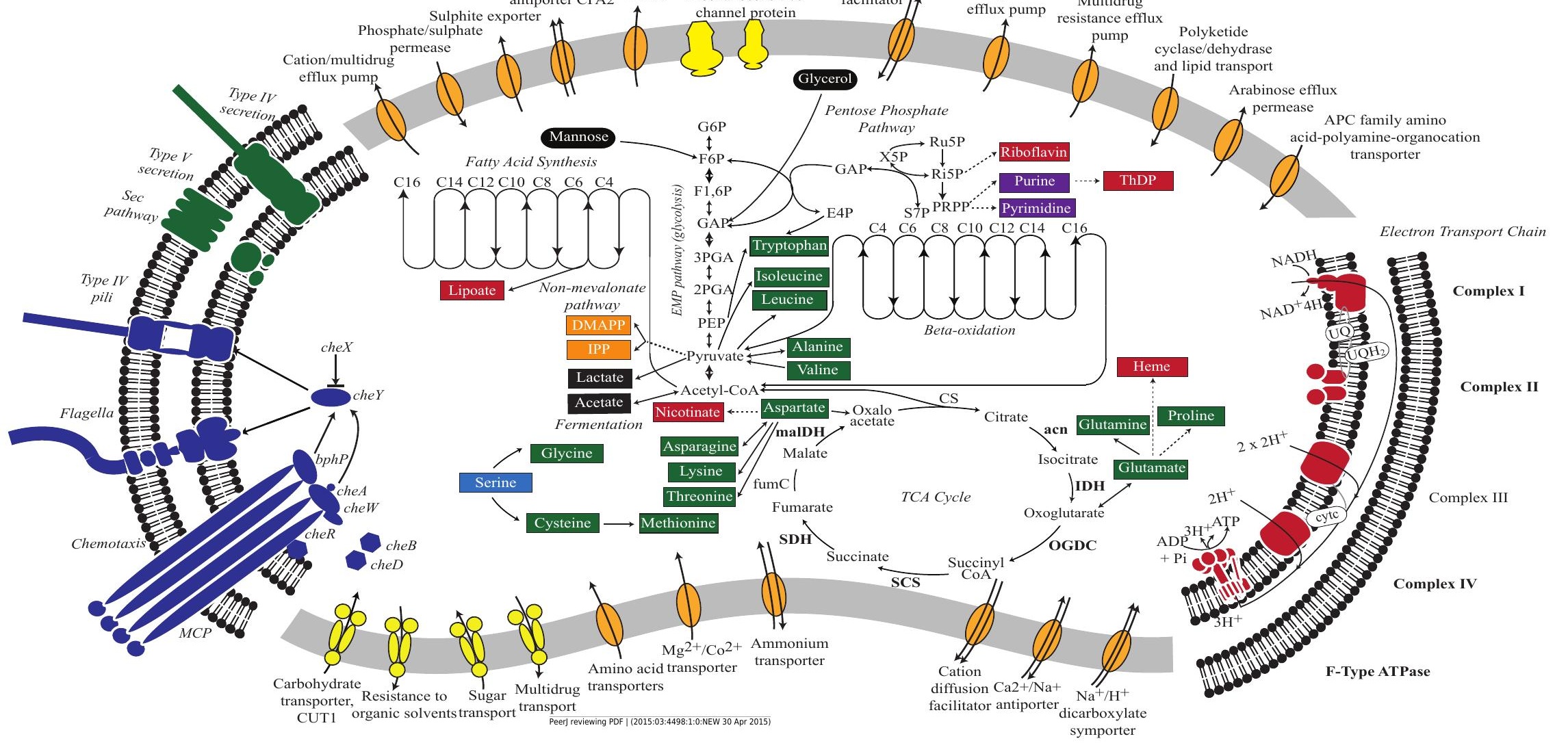


Figure 3 (on next page)

Proposed predatory life cycle of Vampirovibrio chlorellavorus informed by genome annotations

i) V. chlorellavorus seeks out $C$. vulgaris cells via chemotaxis and flagella. ii) it attaches to prey cells via a type IV secretion system (T4SS). iii) plasmid DNA and hydrolytic enzymes are transferred to the prey cells via the T4SS where they degrade algal cell contents (see Fig. 4 for details). iv) algal cell exudates are ingested by $V$. chlorellavorus allowing it to replicate by binary division. v) progeny are released completing the cycle. S - starch granule, $\mathrm{M}$ mitochondria and $\mathrm{N}$ - nucleus. 


\section{Figure 4 (on next page)}

Proposed conjugative mechanism

T4SS operons are found on two conjugative plasmids in V. chlorellavorus. The T-strands of the plasmids are predicted to be made single-stranded by plasmid-encoded relaxases (Fig. S4), nicking the DNA at the origin of transfer and transporting the T-strands to the $C$. vulgaris cell via the mating pair formation. We predict that effector proteins (hydrolytic enzymes) synthesised in the bacterium are also transported via the mating pair formation. The T-strand enters the algal nucleus through a nuclear pore complex and is incorporated into a $C$. vulgaris chromosome. The effector proteins degrade the algae contents which are transported out of the algal cell via T-strand encoded transporters (Fig. S4). The algal lysates are imported into the $V$. chlorellavorus cell providing energy and nutrients for replication. 
PeerJReviewing Manuscript

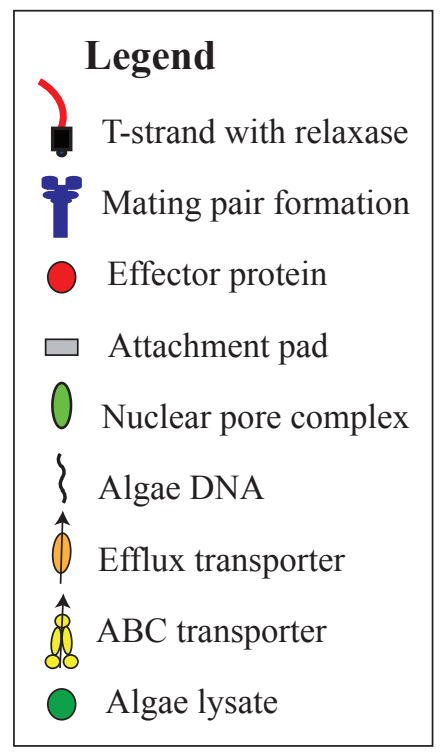

Legend

Effector protein

$\square$ Attachment pad

Nuclear pore complex

Algae DNA

Efflux transporter

\$ ABC transporter

Algae lysate
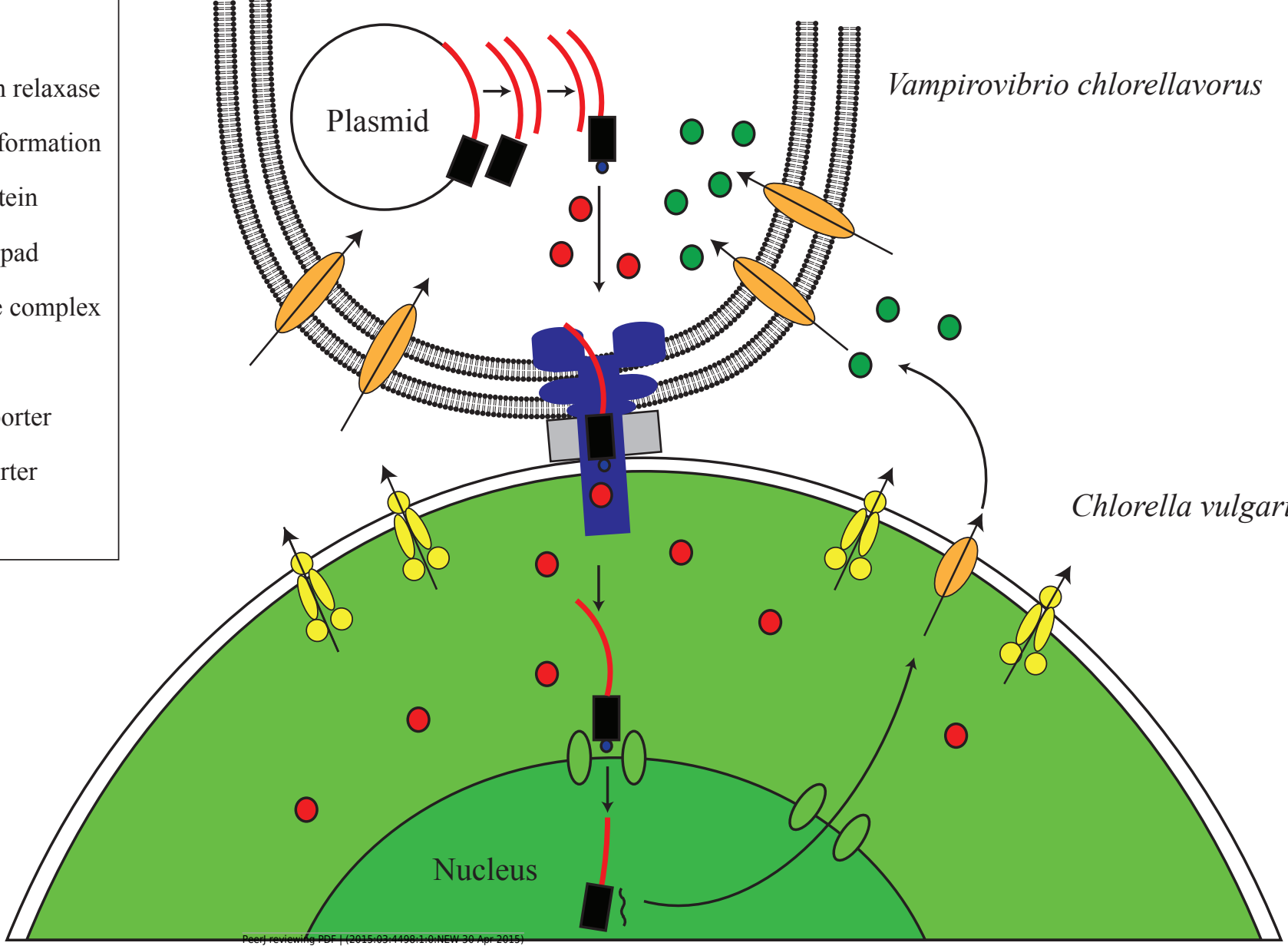
Table 1 (on next page)

Features of the Vampirovibrio chlorellavorus genome

${ }^{a}$ BLASTN search was used to identify the closest neighbour in the May, 2013 version of the Greengenes database (McDonald et al., 2012). ${ }^{\mathrm{b}}$ IslandViewer was used to identify genomic islands (Langille \& Brinkman, 2009) with the SIGI-HMM programme (Waack et al., 2006). c The completeness and contamination of the population genome bin belonging to $V$. chlorellavorus was examined using CheckM v0.9.5 (Parks et al., 2014a). 
2 Table 1. Features of the Vampirovibrio chlorellavorus genome

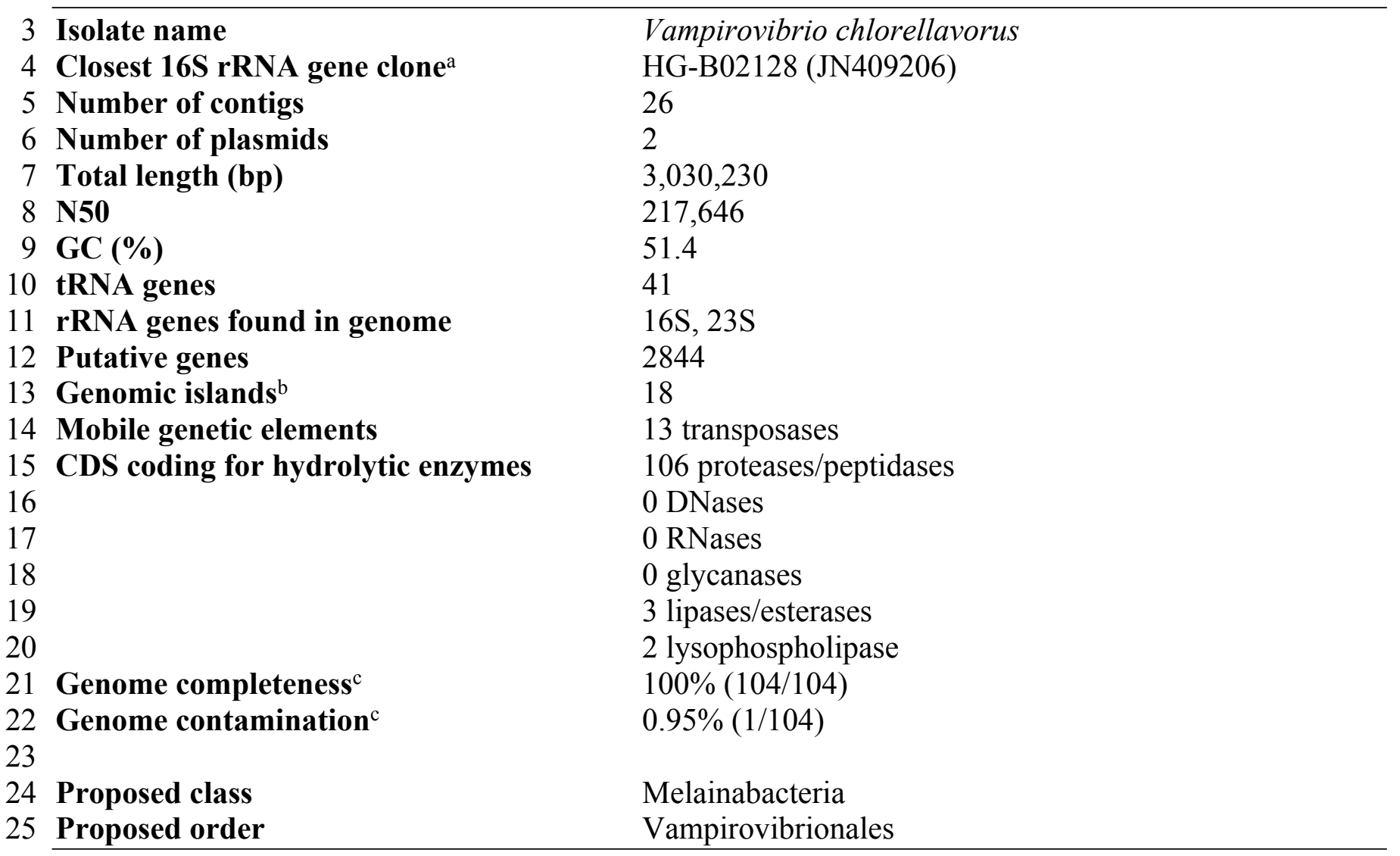

26

27

28

29 